\title{
Correlación entre el Diagnóstico Clínico y Radiográfico de Sujetos Adultos con Erupción Pasiva Alterada
}

\author{
Correlation Between Clinical and Radiographic Diagnosis \\ of Adult Subjects with Altered Passive Eruption
}

César-Augusto Padilla-Avalos; Fabiola Gutiérrez Romero; Consuelo Marroquín-Soto \& Rafael Morales-Vadillo

PADILLA-AVALOS, C. A.; GUTIÉRREZ, R. F.; MARROQUÍN-SOTO, C. \& MORALES-VADILLO, R. Correlación entre el diagnóstico clínico y radiográfico de sujetos adultos con erupción pasiva alterada. Int. J. Odontostomat.,15(3):681-687, 2021.

RESUMEN: El objetivo de este estudio fue determinar la correlación entre el diagnóstico clínico y radiográfico de sujetos adultos con erupción pasiva alterada. La muestra estuvo conformada por los incisivos centrales del cuadrante I de 30 sujetos adultos. Se registraron 2 mediciones clínicas: longitud de corona clínica y distancia del margen gingival (MG) a la unión cemento esmalte (UCE); y 2 mediciones radiográficas: Iongitud de corona anatómica y distancia del MG-UCE. Para diagnosticar EPA, se comparó el promedio de la resta entre corona anatómica y corona clínica; con el valor hipotético ( $\geq 2,5$ mm según Zucchelli). La muestra estuvo conformada por: 36,7 \% incisivos de sujetos de sexo masculino y $63,3 \%$ de sexo femenino; con la edad media de 25,17 años. Clínicamente, se obtuvo la media de 8,5 mm para longitud de corona clínica y 2,267 mm para sondaje MG-UCE. Radiográficamente, se obtuvo la media de 10,767 mm para longitud de la corona radiográfica y 2,47 mm para MG-UCE. Según la Rho de Spearman, el sondaje MG-UCE (clínico) con MG-UCE (radiográfico) no muestra una correlación estadísticamente significativa $(p=0,227)$, sin embargo, al comparar la media $(2,48 \mathrm{~mm})$ con el valor hipotético $(\geq 2,5 \mathrm{~mm})$ según la prueba t de una muestra, se determina que no existe diferencia estadísticamente significativa $(p=0,864)$ entre ambos resultados. La radiografía con técnica paralela de Zucchelli es una excelente alternativa para un adecuado diagnóstico diferencial de la erupción pasiva alterada; de manera simple, económica y reproducible para el beneficio de clínicos y pacientes.

PALABRAS CLAVE: erupción dental, corona del diente, gingival, inserción epitelial.

\section{INTRODUCCIÓN}

Desde hace muchos años, se conoce que la erupción pasiva es el proceso en el cual la adherencia epitelial de los tejidos gingivales migra desde el esmalte hacia apical, ubicándose coronal a la unión cemento esmalte (UCE); este desplazamiento viene acompañado de tejido conectivo de adherencia fibrosa en la base del surco gingival (Gottlieb \& Orban, 1933; Gargiulo et al., 1961). Sin embargo, la erupción pasiva alterada (EPA) es la condición en la cual la relación entre el diente, el hueso alveolar y el tejido blando crea una excesiva exposición gingival (Chan, 2015). Según la Clasificación de Enfermedades y Condiciones Periodontales - Peri implantares 2017, la EPA es una condición del desarrollo, caracterizado por el margen gingival ( $y$, a veces, el hueso) ubicado más coronal y esta relación dentoalveolar anormal se asocia con la formación de pseudobolsas y / o problemas estéticos (Jepsen et al., 2018). La EPA representa una alteración en el desarrollo de la unidad dentogingival por falta de migración apical del margen gingival, produciendo una reducción en la exposición de la corona clínica, siendo una causa de sonrisa gingival (Wilckens et al., 2015; Mostafa et al., 2018; Telletxea et al., 2020).

Existen dos tipos de EPA basados en la relación entre la encía queratinizada y la UCE; y dos subgrupos basados en la relación entre la cresta ósea vestibular y la UCE (Coslet et al., 1977). Se postulan posibles causas relacionadas al origen de la EPA; como: interferencias oclusales por tejidos blandos durante la

Facultad de Odontología, Universidad de San Martín de Porres (FO-USMP), Lima, Perú.

Received: 2021-02-10 Accepted: 2021-05-30 
fase eruptiva y la predisposición hereditaria o familiar (Alpiste-Illueca, 2011). La prevalencia de EPA en población adulta se ha estudiado poco hasta la fecha, debido a la falta de criterios de diagnóstico claros. Al evaluar una población de 1.000 pacientes adultos, con una edad media de 24 años, se informó una prevalencia del 12 \% (Volchansky \& Cleaton-Jones, 1974).

La importancia de diagnosticar correctamente la EPA se justifica porque representa un riesgo para la salud periodontal, que genera dificultades en la higiene bucal, pudiendo causar enfermedad periodontal en pacientes con alta predisposición (Coslet et al.; Alpiste-Illueca, 2011). Asimismo, se argumenta que un surco gingival profundo genera condiciones anaerobias para el desarrollo de gingivitis; por lo tanto, la EPA es un riesgo para los tejidos periodontales que van a recibir restauraciones o coronas (Volchansky \& CleatonJones; Dello Russo, 1984).

La EPA se considera un factor de riesgo potencial para el desarrollo de la enfermedad periodontal y existen varios métodos para diagnosticarla: la exploración clínica de la UCE, la cresta ósea alveolar y los análisis radiográficos (Mele et al., 2018).

Se afirma que no es fácil identificar la UCE a través del sondaje en pacientes con EPA y sólo es eficaz en casos inusuales, ya que comúnmente se percibe una sola interrupción al sondaje y es difícil distinguir la UCE de la cresta ósea. Para el correcto diagnóstico de EPA, se recomienda ampliar el estudio con una radiografía periapical, utilizando la técnica paralela con el uso de paralelizadores de Rinn (dispositivo radiográfico) y mejorada con un marcador radiopaco (Zucchelli, 2013). Del mismo modo, otros autores señalan que el diagnóstico clínico de EPA es incierto por la misma dificultad para distinguir la UCE; y justifica la radiografía con técnica paralela para identificar la UCE (Mele et al.). Dado que el aspecto clínico puede estar sujeto a la percepción de cada profesional (Vassilopoulos et al., 2013), por lo tanto; autores e investigadores proponen la radiografía periapical con la técnica paralela de cono largo en el diagnóstico de EPA (Cairo et al., 2012; Alpiste-lllueca, 2011, 2012; Zucchelli; Rossi \& Brunelli, 2014). Esto debido a que la radiografía periapical con técnica paralela es simple, concisa y reproducible; y permite estudiar las dimensiones del complejo dentogingival en EPA (Alpiste-Illueca, 2004).

Por lo tanto, el presente estudio tuvo como objetivo determinar la correlación entre el diagnóstico clínico y radiográfico de sujetos adultos con erupción pasiva alterada del Centro Odontológico de la Universidad de San Martín de Porres.

El objetivo tuvo como variables: al diagnóstico clínico y radiográfico, para poderse correlacionar. Asimismo, el valor obtenido de la resta entre corona anatómica y clínica fue comparado con el valor hipotético, utilizando la técnica de Zucchelli.

\section{MATERIAL Y MÉTODO}

El diseño metodológico fue de tipo: observacional, descriptivo, prospectivo y transversal. El muestreo fue no probabilístico y la muestra estuvo conformada por los incisivos centrales del cuadrante I de 30 sujetos adultos con erupción pasiva alterada, atendidos en un periodo de tres meses desde el inicio de la ejecución. Para la investigación, se informó a los pacientes sobre el estudio y se pidió su consentimiento informado para poder realizar: un examen periodontal y una toma radiográfica siguiendo el protocolo establecido y siendo aprobado por un Comité de Ética en el Centro Odontológico.

Los criterios de inclusión fueron: pacientes de 20 a 40 años, con una longitud de corona clínica menor igual a $9 \mathrm{~mm}$ en el incisivo central superior del cuadrante I, que además presenten un festoneado gingival excesivamente aplanado y papilas demasiado anchas en relación con su altura (Alpiste-Illueca, 2012).

Los criterios de exclusión fueron: pacientes con enfermedad sistémica, en periodo de embarazo o lactancia y fumadores; o asimismo sean portadores de ortodoncia o prótesis a nivel del incisivo central del cuadrante I, que presente agrandamiento gingival inducido por fármaco, con presencia de sangrado al sondaje o placa al examinar el área; igualmente dientes con UCE no detectable con la sonda periodontal UNC 15 Carolina del Norte o presenten alguna patología a ese nivel. Otros criterios fueron: antecedente de cirugía periodontal (a nivel del diente evaluado) y/o tratamiento de ortodoncia; alteración en el margen incisal, restauración o signos por injuria traumática o atrición.

Calibración. Para la obtención de los datos se realizó una calibración con otros especialistas en el diagnóstico clínico y radiográfico (Coeficiente Kappa 1,0 y 0,98 respectivamente). 
Recolección de los datos. Las mediciones clínicas (longitud de corona clínica y sondaje del UCE MG) fueron realizadas por el propio investigador; y las mediciones radiográficas (longitud de corona anatómica y distancia del UCE MG) fueron realizadas por un Especialista en Radiología Bucal y Maxilofacial.

Diagnóstico Clínico. Para el diagnóstico clínico se ubicó la mitad vestibular del incisivo central del cuadrante I, y se determinaron 2 medidas por cada diente: se registró la longitud en milímetros de la corona clínica (distancia desde el margen incisal hasta el margen gingival) (Fig. 1) y la distancia en milímetros del margen gingival a la UCE. Se realizaron ambas mediciones utilizando la sonda periodontal Carolina del Norte UNC 15 marca Hu-Friedy (Chicago, Estados Unidos).

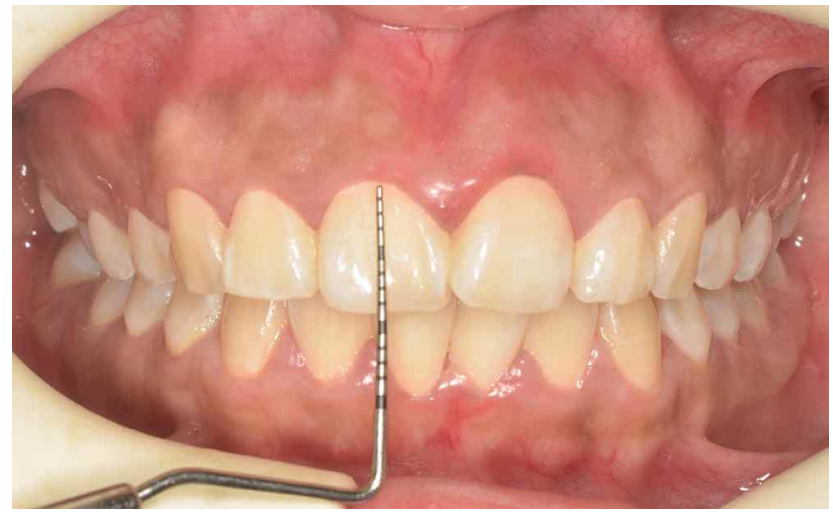

Fig. 1. Medición de la corona clínica en la mitad vestibular del incisivo central del cuadrante I.

Diagnóstico Radiográfico. El equipo radiográfico fue el Plan Meca ProX Intraoral de Plan Meca Helsinki Finlandia, cuya programación es de $63 \mathrm{Kv}$; 6 ma y p160s con las placas de fósforo de marca DÜR DENTAL Bissingen Alemania, (número 2+ Back) como receptores de imagen, las mismas que fueron escaneadas mediante el programa de Scaner VISTA SCAN de DÜR DENTAL (Bissingen Alemania). La radiografía fue realizada con la técnica paralela, empleando un posicionador radiográfico de marca Rinn de Dentsply (Pensilvania, Estados Unidos) (Fig. 2), adicionalmente se utilizó un cono de gutapercha $N^{\circ} 45$ Endomedic de Dentsply (Pensilvania Estados Unidos), (referencia radiopaca que permitió ubicar la posición del margen gingival en la radiografía) colocada desde el margen incisal hasta el margen gingival (corona clínica) mediante una cinta de fijación; en la mitad vestibular del incisivo central del cuadrante I (Fig. 3).

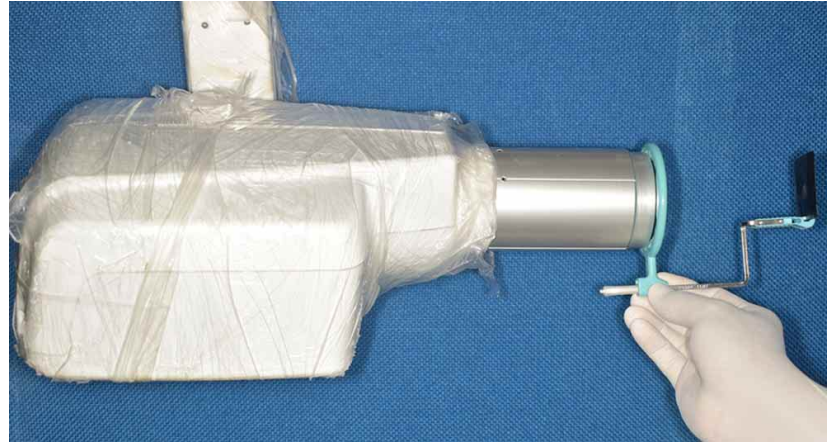

Fig. 2. Equipo radiográfico de cono largo ubicado junto al posicionador radiográfico.

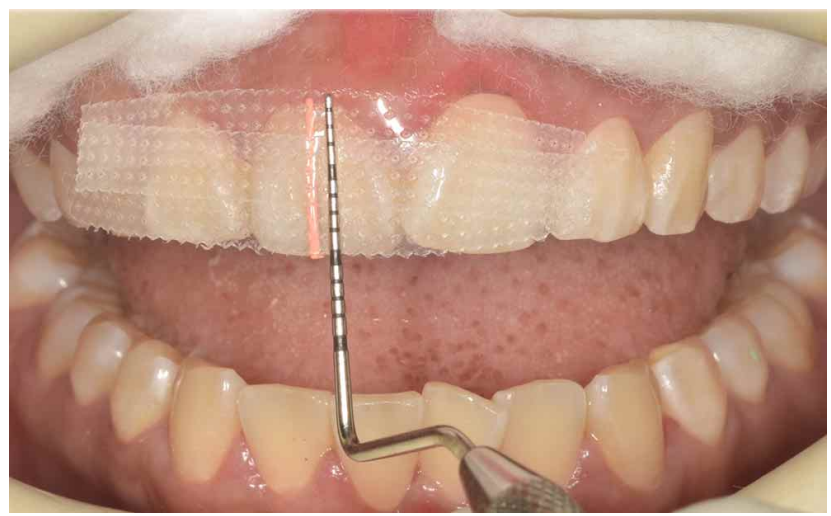

Fig. 3. Colocación del marcador radiopaco en la mitad vestibular del incisivo central del cuadrante I.

Para controlar la distorsión dimensional de imagen, la placa radiográfica fue posicionada paralela al eje axial del diente. Para asegurar una imagen clara, la distancia requerida entre la fuente de rayos $X$ y el diente fue de $20 \mathrm{~cm}$. El haz de rayos estuvo orientado para que el haz central incida perpendicularmente hacia el eje del diente y la placa. Para evitar la distorsión en sentido vertical, se verificó la medida de la corona clínica (margen incisal al margen gingival) con la medida del marcador radiopaco; y para evitar la distorsión en sentido horizontal, se ubicó el centro del aro del posicionador radiográfico a nivel del incisivo central del cuadrante I de cada paciente.

Se determinaron 2 medidas radiográficas de incisivo central del cuadrante I: la longitud de la corona radiográfica (margen incisal a la UCE) y la distancia del margen gingival a la UCE). La posición del margen gingival fue revelada por el marcador radiopaco (Fig. 4).

Las mediciones clínicas fueron valores enteros (instrumento: sonda Carolina del Norte UNC 15); mientras que la medición imagenológica fue en escala real mediante la radiografía digital. El promedio de la resta 


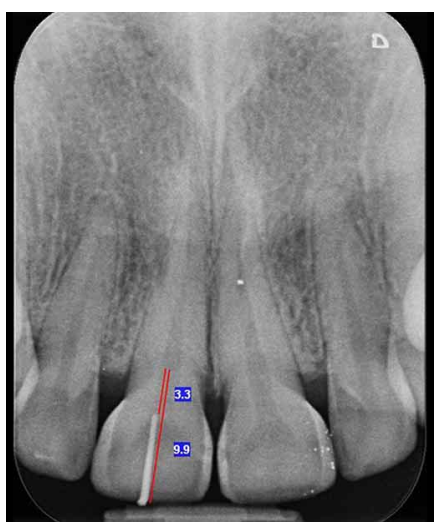

Fig. 4 Radiografía periapical del incisivo central del cuadrante I.

Longitud de la corona anatómica $(9.9 \mathrm{~mm})$. El marcador radiopaco (gutapercha) evidencia la posición del margen gingival, por lo tanto, la distancia del MG al UCE es $3.3 \mathrm{~mm}$.

de corona anatómica y corona clínica fue comparado con un referente hipotético ( $\geq 2,5 \mathrm{~mm}$ para erupción pasiva alterada), (Zucchelli) (Fig. 5).

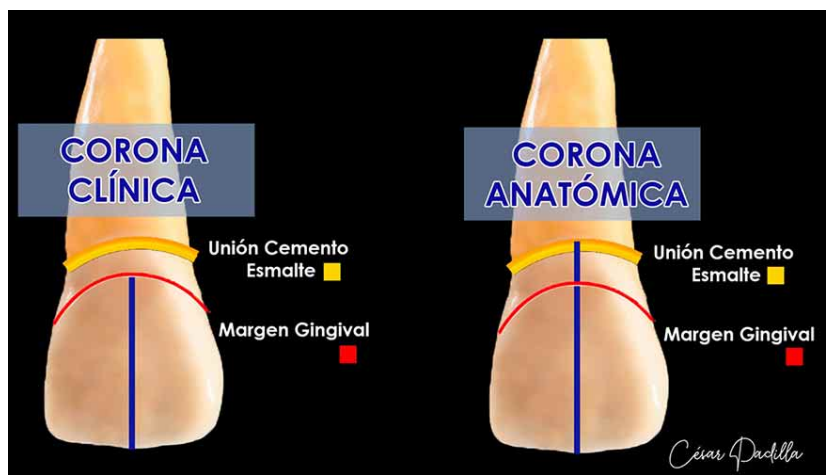

Fig. 5. Referencias anatómicas para el diagnóstico de EPA.

El análisis estadístico se realizó en el software SPSS. Todos los valores de cálculo inferencial fueron considerados con significancia estadística cuando se ubicaban por debajo del $0,05(p<0,05)$. Las medidas clínicas y radiográficas se correlacionaron mediante la Prueba no paramétrica de Spearman.

\section{RESULTADOS}

La distribución por sexo en pacientes con EPA, tuvo la frecuencia de $11(36,7 \%)$ y $19(63,3 \%)$ para sexo masculino y femenino respectivamente; para la edad se obtuvo una media de 25,17 y mediana de 25 años.

Para determinar el diagnóstico clínico de EPA; los datos de longitud de corona clínica y sondaje MG UCE, fueron analizados mediante la prueba de Shapiro-Wilk y los datos no mostraron semejanza a la distribución normal $(p<0,001)$. Se obtuvo la media de $8,5 \mathrm{~mm}$ y mediana de $9 \mathrm{~mm}$ para longitud de corona clínica; y para sondaje MG UCE, la media de 2,267 $\mathrm{mm}$ y mediana de $2 \mathrm{~mm}$ (Tabla I).

Para determinar el diagnóstico radiográfico de EPA; los datos de longitud de corona anatómica y MG UCE (radiográfico), fueron analizados mediante la prueba de Shapiro-Wilk y los datos no mostraron semejanza a la distribución normal $(p<0,001)$. Se obtuvo la media de $10,767 \mathrm{~mm}$ y mediana de $11 \mathrm{~mm}$ para longitud de corona anatómica; y para MG UCE (radiográfico), la media de 2,47 mm y mediana de 2,45 $\mathrm{mm}$ (Tabla II).

Según la Rho de Spearman, se determinó que el Sondaje MG UCE (medición clínico) con MG UCE (medición radiográfico), no muestra una correlación estadísticamente significativa $(p=0,227)$.

Los datos obtenidos de EPA (Diferencia de Corona Anatómica - Corona Clínica) fueron analizados mediante la prueba de Shapiro-Wilk y se observó semejanza a la distribución normal en los datos recolec-

Tabla I. Diagnóstico Clínico (Longitud de Corona Clínica y Sondaje MG UCE).

\begin{tabular}{llcc}
\hline Longitud de Corona & Media & & 8.500 \\
Clínica & $95 \%$ de intervalo de confianza para la & Límite inferior & 8.245 \\
& media & Límite superior & 8.755 \\
& Mediana & & 9.000 \\
& Desviación & 0.6823 & 7.0 \\
& Mínimo & 9.0 & 2.267 \\
Sóximo & Media & & 2.099 \\
& $95 \%$ de intervalo de confianza para la & Límite inferior & 2.435 \\
& media & Límite superior & 2.000 \\
& Mediana & & 0.4498 \\
& Desviación & & 2.0 \\
& Mínimo & & 3.0
\end{tabular}


Tabla II. Diagnóstico Radiográfico (Longitud de Corona Anatómica y MG UCE).

\begin{tabular}{|c|c|c|c|}
\hline \multirow{7}{*}{ Anatómica } & Media & & 10.767 \\
\hline & \multirow{2}{*}{$\begin{array}{l}95 \% \text { de intervalo de confianza para la } \\
\text { media }\end{array}$} & Límite inferior & 10.554 \\
\hline & & Límite superior & 10.979 \\
\hline & \multicolumn{2}{|l|}{ Mediana } & 11.000 \\
\hline & \multicolumn{2}{|l|}{ Desviación } & 0.5683 \\
\hline & \multicolumn{2}{|l|}{ Mínimo } & 10.0 \\
\hline & \multicolumn{2}{|l|}{ Máximo } & 12.0 \\
\hline \multirow[t]{7}{*}{ MG UCE } & Media & & 2.470 \\
\hline & \multirow{2}{*}{$\begin{array}{l}95 \% \text { de intervalo de confianza para la } \\
\text { media }\end{array}$} & Límite inferior & 2.132 \\
\hline & & Límite superior & 2.808 \\
\hline & Mediana & & 2.450 \\
\hline & Desviación & & 0.9041 \\
\hline & Mínimo & & 0.3 \\
\hline & Máximo & & 4.6 \\
\hline
\end{tabular}

tados (Prueba de Shapiro-Wilk: $p=0,076)$, se obtuvo la media de $2,48 \mathrm{~mm}$ y mediana de $2,6 \mathrm{~mm}$ para el valor de EPA.

Para determinar EPA, el valor debe ser mayor igual a 2,5, entonces se utilizó la prueba t de una muestra para comparar la media de las observaciones $(2,6)$ con el valor hipotético. Concluyendo que no existe diferencia estadísticamente significativa entre los valores analizados $(p=0,864)$.

\section{DISCUSIÓN}

La erupción pasiva alterada (migración apical incompleta del margen gingival en el adulto) es la causa más común de coronas cortas; siendo ésta, la principal solicitud de tratamiento, debido al problema estético asociado a la excesiva exposición del tejido gingival. Esta condición, produce un difícil control de placa (debido al exceso gingival), inflamación gingival y formación de pseudobolsas (aumento de profundidad de sondaje sin pérdida de inserción) (Zucchelli).

Para el diagnóstico de EPA, se ha determinado dos criterios subjetivos: el festoneado excesivamente aplanado y las papilas demasiado anchas en relación con su altura; en coronas clínicas cortas (Alpiste-Illueca, 2012). Por otra parte, se determina corona clínica corta si el valor es inferior a $8 \mathrm{~mm}$ en el incisivo central superior (Monaco et al., 2004), y en otros estudios cuando es menor a $9 \mathrm{~mm}$ de longitud (Robbins, 1999). Para el presente estudio, se evaluaron 30 incisivos centrales del cuadrante I, considerando los criterios diagnósticos establecidos por los autores (Alpiste-Illueca, 2004), y se obtuvo una media de $8,5 \mathrm{~mm}$ y mediana de $9 \mathrm{~mm}$ para longitud de corona clínica (margen incisal al margen gingival). Esto debido a la estrecha relación que existe entre el excesivo festoneado gingival con papilas demasiado anchas, en coronas clínicas cortas.

Se ha señalado que, en el adulto joven, el margen gingival se encuentra $1 \mathrm{~mm}$ coronal al UCE; por lo tanto, es importante registrar esta distancia para anticipar la futura posición del margen gingival (Ramzi \& Nada, 2013). Se considera fundamental la detección del UCE en el sondaje para el diagnóstico diferencial, sin embargo, esta detección (en pacientes con EPA) no es sencilla por dos fundamentos: la encía vestibular por lo general presenta una mayor adherencia a la superficie del esmalte; y la cresta ósea vestibular se encuentra a nivel más coronal al UCE (Zucchelli). Tomando en consideración dichas investigaciones; el presente estudio determinó clínicamente la distancia del margen gingival a la UCE (en pacientes con EPA) en el sondaje del incisivo central superior y se obtuvo una media de $2,267 \mathrm{~mm}$ y mediana de $2 \mathrm{~mm}$. Estas mediciones podrían estar sujetas a variabilidad debido a las dificultades clínicas ya descritas con anterioridad (Zucchelli).

En un estudio, se correlacionó la evaluación clínica con la medición radiográfica para determinar la altura de la corona clínica (Cairo et al.). Asímismo se indicó que el tratamiento de EPA inicia con una evaluación de la sonrisa, ayudada por las reglas de Chu que evalúan la proporción estética de los dientes antero superiores, radiografías periapicales para medir y superponer la situación clínica a la corona "oculta" (Rossi \& Brunelli).

Con anterioridad, la unidad dentogingival del incisivo central superior, se han evaluado clínica y radiográficamente; asimismo se desarrolló una técnica 
radiográfica para medir la unidad dentogingival; utilizando un cono de gutapercha en la base del surco. Justificando que la técnica radiográfica es simple, reproducible, concisa y de gran ayuda para el diagnóstico de EPA (Alpiste-Illueca, 2004, 2012). Se evidenció que las mediciones de Sondaje MG UCE (medición clínica) con MG UCE (medición radiográfico); no muestran una correlación estadísticamente significativa $(p=0,227)$. Esto indica que existen limitaciones en la detección clínica del MG UCE, afirmando la dificultad que expresa Zucchelli lo que sugiere ampliar el estudio con una radiografía de técnica paralela.

Existen antecedentes, que determinan el diagnóstico de EPA, con la diferencia entre longitud de corona clínica y corona anatómica (radiográfica). Incluso, se sugiere el sondaje periodontal para identificar la UCE y medir la corona anatómica; al comparar ambas mediciones, se puede determinar si las coronas clínicas cortas se deben a un desgaste incisal excesivo o a EPA (Balda García et al., 2006) Estas teorías han sido reforzadas, cuando se afirma una EPA verdadera, cuando existe en una notable diferencia $>3 \mathrm{~mm}$ entre la longitud de la corona clínica del diente (distancia del margen incisal al margen gingival) y la corona radiográfica (distancia del margen incisal al UCE) (Zucchelli).

Por lo tanto, según Zucchelli, para diagnosticar EPA en el presente estudio; se concluye que no existe diferencia estadísticamente significativa entre los valores analizados $(p=0,864)$ validando la técnica de diagnóstico desarrollada (Zucchelli).

Otros autores han utilizado tomografía computarizada Cone Bean (CBCT) indicando que ésta también podría utilizarse en el diagnóstico y plan del tratamiento de EPA (Batista Jr. et al., 2012). Sin embargo, autores creen que las radiografías periapicales que se utilizan con un marcador radiopaco pueden ser menos invasivo para detectar de manera aproximada la presencia o ausencia de una alteración en la erupción pasiva (Pavone et al., 2016). La presente investigación al ser prospectiva, limitaba el uso de CBCT por razones éticas. Además, que la técnica desarrollada plantea una alternativa al equipo tomógrafo, dado que éste no siempre está accesible en la consulta cotidiana.

Según una exhaustiva búsqueda de la literatura, cabe resaltar que no existe ningún trabajo de investigación con la misma metodología; por lo tanto, el presente estudio ha desarrollado la técnica de Zucchelli; con originalidad, lo cual limita la discusión de resultados o hallazgos con los demás autores.

\section{CONCLUSIONES}

En el diagnóstico clínico de EPA se obtuvo la media de $8,5 \mathrm{~mm}$ para longitud de corona clínica y 2,267 mm para el sondaje MG UCE. En el diagnóstico radiográfico de EPA se obtuvo la media de $10.767 \mathrm{~mm}$ para longitud de corona radiográfica y 2,47 para MG UCE (medición radiográfica). Se evidenció que no existe correlación estadísticamente significativa entre el diagnóstico clínico y radiográfico (Prueba Rho de Spearman $p=0,227$ ).Para los datos de EPA (Resta de corona anatómica - corona clínica), se obtuvo la media de $2,48 \mathrm{~mm}$ y mediana de $2,6 \mathrm{~mm}$.

Entonces, para diagnosticar EPA según Zucchelli, la media $(2,48 \mathrm{~mm})$ se comparó con el valor hipotético (mayor igual a $2,5 \mathrm{~mm}$ ), determinando que no existe diferencia estadísticamente significativa $(p=0,864)$. Por lo que se concluye, que la técnica de Zucchelli es una alternativa para el diagnóstico de la erupción pasiva alterada.

El diagnóstico clínico de la erupción pasiva alterada presenta limitaciones, y aunque se han desarrollado diversos métodos, existen criterios poco definidos; por lo tanto, es primordial desarrollar técnicas complementarias en radiología para correlacionar ambos diagnósticos.

La radiografía con la técnica paralela de Zucchelli mejorada con un marcador radiopaco, es una excelente alternativa para el diagnóstico de la erupción pasiva alterada.

La investigación brinda los métodos para un adecuado diagnóstico diferencial de la erupción pasiva alterada, de manera simple, económica y reproducible para el beneficio de todos los pacientes.

PADILLA-AVALOS, C. A.; GUTIÉRREZ, R. F.; MARROQUÍN-SOTO, C. \& MORALES-VADILLO, R. Correlation between clinical and radiographic diagnosis of adult subjects with altered passive eruption. Int. J. Odontostomat., 15(3):681-687, 2021.

ABSTRACT: The aim of the study was to determine the correlation between the clinical and radiographic diagnosis of adult subjects with altered passive eruption. The population consisted of the central incisors of quadrant I of 30 adult subjects. Two clinical measurements were recorded: length of clinical crown and distance from the gingival margin (GM) to the cementum enamel junction (CEJ); and 2 radiographic measurements: length of anatomical crown and 
distance from the GM-CEJ. To diagnose APE, the average of the subtraction between anatomical crown and clinical crown was compared; with the hypothetical value $(\geq 2.5 \mathrm{~mm}$ according to Zucchelli). The population were: $36.7 \%$ incisors of male subjects and $63.3 \%$ female; with the average age of 25.17 years. Clinically, the average was $8.5 \mathrm{~mm}$ for clinical crown length and 2,267 $\mathrm{mm}$ for GM-CEJ probing. Radiographically, the average $10.767 \mathrm{~mm}$ was obtained for the length of the radiographic crown and $2.47 \mathrm{~mm}$ for GMCEJ. According to Spearman's Rho, the GM-CEJ (clinical) probing with GM-CEJ (radiographic) does not show a statistically significant correlation $(p=0.227)$, however, when comparing the average $(2.48 \mathrm{~mm})$ with the hypothetical value ( $\geq 2.5 \mathrm{~mm}$ ) according to the t-test of a sample, it is determined that there is no statistically significant difference $(p=0.864)$ between both results. The radiography with Zucchelli technique is an excellent alternative for an adequate differential diagnosis of altered passive eruption; in a simple, economical and reproducible way for the benefit of clinicians and patients.

KEY WORDS: dental eruption, dental crown, gingival, epithelial attachment.

\section{REFERENCIAS BIBLIOGRÁFICAS}

Alpiste-lllueca, F. Altered passive eruption (APE): A little known clinical situation. Med. Oral Patol. Oral Cir. Bucal, 16(1):e100-4, 2011.

Alpiste-Illueca, F. Dimensions of the dentogingival unit in maxillary anterior teeth: a new exploration technique (parallel profile radiograph). Int. J. Periodontics Restorative Dent., 24(4):38696, 2004.

Alpiste-lllueca, F. Morphology and dimensions of the dentogingival unit in the altered passive eruption. Med. Oral Patol. Oral Cir. Bucal, 17(5):e814-20, 2012.

Balda García, I.; Herrera Ureña, J. I.; Frías López, M. C. \& Carasol Campillo, M. Erupción pasiva alterada. Implicaciones estéticas y alternativas terapéuticas. R. C. O. E., 11(5-6):563-71, 2006.

Batista Jr., E. L.; Moreira, C.; Batista, F. C.; de Oliveira, R. R. \& Pereira, K. K. Y. Altered passive eruption diagnosis and treatment: a cone beam computed tomography-based reappraisal of the condition. J. Clin. Periodontol., 39(11):1089-96, 2012.

Cairo, F.; Graziani, F.; Franchi, L.; Defraia, E. \& Pini Prato, G. P. Periodontal plastic surgery to improve aesthetics in patients with altered passive eruption/gummy smile: a case series study. Int. J. Dent., 2012:837658, 2012.

Chan, D. K. Predictable treatment for "Gummy Smiles" due to altered passive eruption. Inside Dent.,11(7), 2015.

Coslet, G. J.; Vanarsdall, R. \& Weisgold, A. Diagnosis and classification of delayed passive eruption of the dentogingival junction in the adult. Alpha Omegan, 70(3):24-8, 1977.

Dello Russo, N. M. Placement of crown margins in patients with altered passive eruption. Int. J. Periodontics Restorative Dent., 4(1):58-65, 1984.

Gargiulo, A. W.; Wentz, F. \& Orban, B. Dimension and relations of the dentogingival junction in humans. J. Periodontol., 32(3):2617, 1961.

Gottlieb, B. \& Orban, B. Active and passive continuous eruption teeth. J. Dent. Res., 13:214, 1933.

Jepsen, S.; Caton, J. G.; Albandar, J. M.; Bissada, N. F.; Bouchard, P.; Cortellini, P.; Demirel, K.; de Sanctis, M.; Ercoli, C.; Fan, J.; et al. Periodontal manifestations of systemic diseases and developmental and acquired conditions: Consensus report of workgroup 3 of the 2017 World Workshop on the Classification of Periodontal and Peri-Implant Diseases and Conditions. J. Clin. Periodontol., 89 Suppl. 1:S237-S248, 2018.

Mele, M.; Felice, P.; Sharma, P.; Mazzotti, C.; Bellone, P. \& Zucchelli, G. Esthetic treatment of altered passive eruption. Periodontol. 2000, 77(1):65-83, 2018.

Monaco, A.; Streni, O.; Marci, M. C.; Marzo, G.; Gatto, R. \& Giannoni, M. Gummy smile: clinical parameters useful for diagnosis and therapeutical approach. J. Clin. Pediatr. Dent., 29(1):19-25, 2004.

Mostafa, D. A successful management of sever gummy smile using gingivectomy and botulinum toxin injection: A case report. Int. J. Surg. Case Rep., 42:169-74, 2018.

Pavone, A. F.; Ghassemian, M. \& Verardi, S. Gummy Smile and Short Tooth Syndrome--Part 1: Etiopathogenesis, Classification, and Diagnostic Guidelines. Compend. Contin. Educ. Dent., 37(2):102-7, 2016.

Ramzi, V. \& Nada, M. Periodontal treatment of excessive gingival display. Semin. Orhod., 19(4):267-78, 2013.

Robbins, J. W. Differential diagnosis and treatment of excess gingival display. Pract. Periodont. Aesthet. Dent., 11(2):265-72, 1999.

Rossi, R. \& Brunelli, G. Altered passive eruption and familial trait: a preliminary investigation. Int. J. Dent., 2014:874092, 2014.

Telletxea, I. M.; López-Malla, M. J. \& Alía, G. E. Las tres caras de la sonrisa gingival. Revisión bibliográfica. Cient. Dent., 17(2):13946, 2020.

Vassilopoulos, P. J.; Geurs, N. C. \& Geisinger, M. L. Parameters that influence the position of the facial gingival margin on maxillary anterior teeth. Semin. Orthod., 19(1):37-48, 2013.

Volchansky, A. \& Cleaton-Jones, P. E. Delayed passive eruption. A predisposing factor to Vincent's infection. J. Dent. Ass. S. Afr., 29(5):291-4, 1974.

Wilckens, M.; Beltrán, V.; Leivac, C. \& Donaire, F. Manejo quirúrgico periodontal de la erupción pasiva alterada: reporte de casos. Rev. Clin. Periodoncia Implantol. Rehabil. Oral, 8(2):167-72, 2015.

Zucchelli, G. Erupción Pasiva Alterada. En: Gori, G. (Ed.). Cirugía Estética Mucogingival. Berlin, Quintessence Publishing, 2013. pp.749-93.

Dirección para correspondencia:

César-Augusto Padilla-Avalos

Maestría en Periodoncia

Facultad de Odontología

Universidad de San Martín de Porres (FO-USMP)

Lima - PERÚ

Email: cesarpadilla160@gmail.com

César Padilla-Avalos

Fabiola Gutiérrez Romero

Consuelo Marroquín-Soto

Rafael Morales-Vadillo orcid.org/0000-0002-8436-4113

orcid.org/0000-0002-1929-0306

orcid.org/0000-0002-1433-6205

orcid.org/0000-0002-7835-6408 